Science, 1972, 175, pp. 1020-1022.

\title{
Mapping of Interactions in the Pitch Memory Store
}

\begin{abstract}
A technique obtaining a precise mapping of interactive effects in the pitch memory store is described. Subjects were required to compare two tones for pitch when these were separated by a 5-second interval during which six other tones were played. In the second serial position of the intervening sequence there was placed a tone whose pitch bore a critical relationship to the pitch of the first test tone. When the critical intervening tone was identical in pitch to the first test tone, memory facilitation was produced. As the separation in pitch between these two tones increased, errors rose progressively, peaked at a separation of 2/3 tone, and declined roughly to baseline at a whole tone separation. It is concluded that the pitch memory store is arranged logarithmically in a highly ordered and specific fashion.
\end{abstract}

Investigations into human memory storage have traditionally emphasized the use of verbal materials; in contrast little is known about the retention of unlabeled sensory stimuli. Yet in exploring a memory store there are obvious advantages to the use of stimuli which can be precisely specified and controlled, and which can be varied along one dimension at a time. Tonal pitch represents an example of such a stimulus. The experiment reported here demonstrates that the pitch memory store is laid out in a highly ordered and specific fashion, and that is possible to map very precisely both facilitatory and disruptive interactions taking place within it.

Previous studies have shown that pitch information decays spontaneously though slowly in the absence of intervening stimulation (1). The incorporation of other tones during the retention interval produces considerable memory disruption $(2,3)$. This interference cannot be explained in such general terms as a limitation in general short-term memory capacity, or a distraction of attention, since the incorporation during the retention interval of spoken numbers (which the subjects are required later to recall) produces only a minimal decrement in the same pitch recognition task that is severely disrupted by the interpolation of other tones (3). It is clear, therefore, that interactive effects must take place within the pitch memory store itself. The following study represents a mapping of such effects.

Subjects were required to make a series of judgments of the following nature. A 200-msec test tone was played, which was followed 5 seconds later by a second 200-msec test tone. During the retention interval six other tones were played. These were also $200 \mathrm{msec}$ in duration, and were separated by 300 -msec intervals, leaving a 2 -second pause before the second test tone. The subjects were instructed to ignore the intervening tones, and simply to indicate whether the test tones were the same or different in pitch by writing "S" or "D."

For the test tone stimuli 12 tonal pitches were used. These were taken from an equal-tempered scale, and spanned an octave range from middle $\mathrm{C}$ to the $\mathrm{B}$ above. For the intervening tones, 24 tonal pitches were used. These were taken from the same scale and spanned a two-octave range from the $\mathrm{F \#}$ below middle $\mathrm{C}$ to the $\mathrm{F}$ about an octave and half above (4).

The experiment consisted of eight conditions. In all conditions but the last, there was placed in the second serial position of the intervening sequence a tone whose pitch bore a critical relationship to the pitch of the first test tone. This relationship varied from that of identity to that of a whole-tone separation in the equal-tempered scale (4). For each of these seven conditions a unique value of pitch separation was incorporated. These values were placed at equal intervals of $1 / 6$ tone within this whole-tone range. Since the musical scale is logarithmic, these intervals were also logarithmic. In the eighth condition, no such critical tone was incorporated. Instead, the pitch of the tone in the second serial position was chosen in the same way as were the pitches of the other tones in the intervening sequence (4). This "null condition" thus provided a baseline for the assessment of the effects of the critical tones.

For each condition, 12 sequences were presented, and the same 12 combinations of test tone pitches were employed in all conditions. The entire set of sequences was presented in random order in groups of 12 , with 10 -second pauses between sequences 
and 2-minute pauses between groups of sequences. Subjects listened to the entire tape on two separate occasions, and the results were averaged.

Tones were generated by a Wavetek oscillator controlled by a PDP 9 computer and were recorded on tape (5). Twelve subjects were used, selected on the basis of obtaining a score of at least 90 percent correct on a short tape containing sequences designed as in the "null" condition.

The effects of inclusion of tones bearing specific relationships to be pitches of the first test tones are shown in Figs. 1 and 2. It can be seen that these effects can be mapped very precisely as a function of these tonal relationships. When the critical included tone is identical in pitch to the first test tone there results a statistically significant decrease in errors compared with the null condition $(P=.02$, two-tailed on a Wilcoxon test). As the difference in pitch between the first test tone and the critical tone is increased, errors rise systematically and peak at a

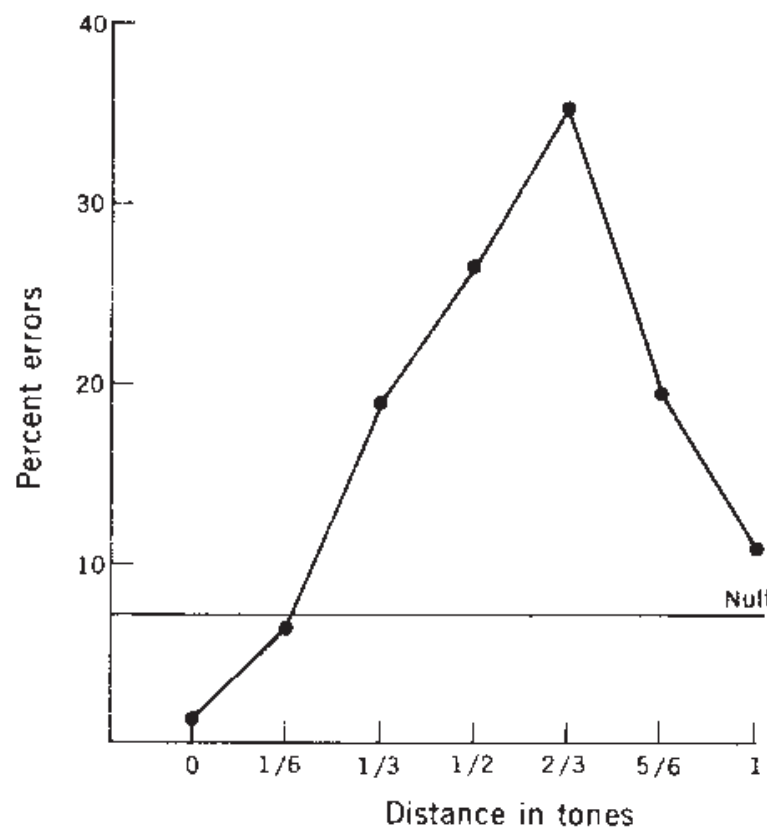

Fig. 1. The percentage of errors in pitch comparisons plotted as a function of the separation in pitch between the critical interpolated tone and the first test tone. The line labeled Null shows the number of errors in the control condition where no tone closer in pitch to the first test tone than $1 \frac{1}{2}$ tones was included in the intervening sequence. A separation of 1/6 tone, in the range of pitches used here, is equal to $5 \mathrm{cycle} / \mathrm{sec}$ at the lowest test tone pitches employed, and $9 \mathrm{cycle} / \mathrm{sec}$ at the highest test tone pitches employed.

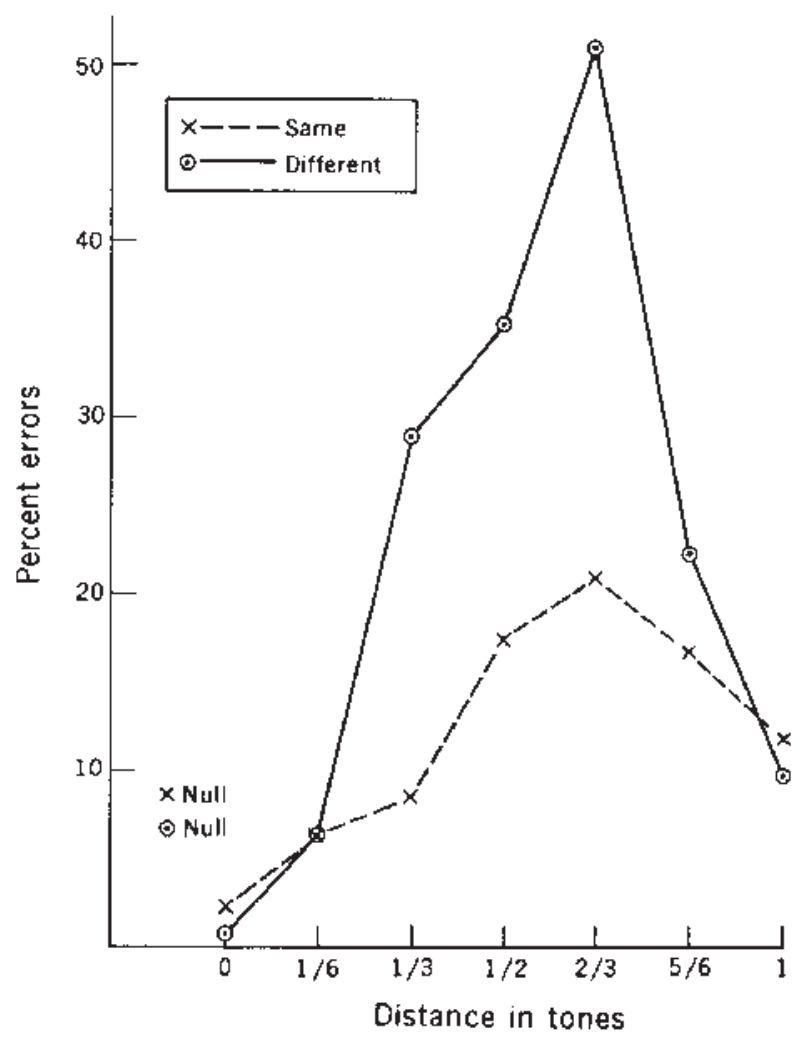

Fig. 2. The percentage of errors in pitch comparisons plotted as in Fig. 1, but separately by whether the test tones were the same or different in pitch. The two Null points indicated refer to the same control condition as described in Fig. 1.

separation of $2 / 3$ tone, declining to roughly the same value as in the null condition when a wholetone separation is reached. The increase in errors produced by the critical tone, compared with the null condition, is statistically significant for all values from $1 / 3$ tone to $5 / 6$ tone separation (in each case $P<.01$, two tailed on a Wilcoxon test).

The U-shaped function obtained here is not due to response bias, as is shown in Fig. 2. Here, errors are plotted separately by whether the test tones are the same in pitch or whether they are different. It can be seen that the characteristics described above are present for both functions. However, errors rise more steeply and are more sharply peaked when the test tones differ in pitch than when they are the same. Further, when the test tones differ in pitch, the increment in errors compared with the null condition is significant for all values from $1 / 3$ tone to $5 / 6$ tone separation $(P<.01$, two-tailed on a Wilcoxon test for each value). But when the test tones are identical in pitch a significant increment in 
errors (measured on the same test) occurs only at $1 / 2$ tone $(P<.05)$ and at $2 / 3$ tone $(P<.01)$ separation. It remains to be demonstrated that the two functions displayed in Fig. 2 are produced by the same underlying process.

The facilitatory and inhibitory interactions displayed here may be related to those obtained in traditional studies on the effect of similarity of interpolated materials on long-term memory for verbal items. Several such studies have also found errors to be a non-monotonic function of similarity between the test items and interpolated items, with an initial facilitatory component to the curve (6). From a different point of view, the findings obtained here may be related to demonstrations of lateral inhibition in various sensory systems, including neural pathways relaying pitch information (7).

A further point of interest concerns the precision with which it is possible to superimpose the results obtained from sequences with test tones taken from different positions in the pitch scale. Since the musical scale is logarithmic, an identical musical interval represents an increased difference in cycles per second as the scale is ascended. This difference doubles at each octave, and so virtually doubles over the range employed in this experiment. Therefore, if the pitch memory store were organized in any fashion other than logarithmic, one would expect a systematic shift in peak of errors as the test tone combinations shift in their position along the scale. For instance, if the pitch memory store were laid out in a linear fashion, the peak of errors should appear to move progressively closer to the pitch of the first test tone as the test tone combinations move upward in scale. However, no such peak shift in either direction is discernible from the data. It would therefore appear that the pitch memory store is logarithmically arranged.

DIANA DEUTSCH

Center for Human Information Processing, University of California, San Diego 92037

\section{References and Notes}

1 J. D. Harris, J. Exp. Psychol. 43, 96 (1952).

2 W. A. Wickelgren, ibid. 72, 250 (1966); J. Math. Psychol. 6, 13 (1969).

3 D. Deutsch, Science 168, 1604 (1970).

4 The tonal pitches were taken from an equal-tempered scale (International Pitch; $A=435$ ). This is arranged in semitone steps and the frequencies employed in this experiment were as follows: F\#(183), G(194), G\#(205), A(218), A\#(230), B(244), C(259), C\#(274), D(290), D\#(308), E(326), F(345), F\#(366), G(388), G\#(411), A(435), A\#(461), B(488), C(517), C\#(548), D(581),

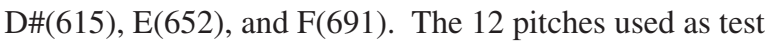
tones ranged from $\mathrm{C}(259)$ to $\mathrm{B}(488)$. In half of the sequences the test tones were the same in pitch, and the other half they differed by a semitone (in the upward direction in half of the instances and in the downward direction in the other half). When the test tones differed in pitch, the critical interpolated tone was placed on the same side along the pitch dimension as was the second test tone. Thus this critical tone was higher than the first test tone in half of these sequences and lower in the other half. This was also the case when the test tones were identical in pitch. For the intervening tones, the 24 tonal pitches ranging from $F \#(183)$ to $F(691)$ were all employed. No two tones which were identical in pitch or were separated by exactly an octave were incorporated in any one sequence. Further, in any sequence all tonal pitches were excluded which lay within, and including, a whole-tone range from the first test tone in either direction (or which were displaced by an octave from this range). This gap in the span of intervening tonal pitches was necessary to prevent the random inclusion of tones in the critical range under study. Apart from these restrictions, the intervening tonal pitches were chosen randomly from the two-octave span described above.

5 All tones were recorded at equal amplitude, and the gain on the tape amplifier was so adjusted that the different tonal pitches appeared equally loud to my ear.

6 E. R. Hilgard and G. H. Bower, Theories of Learning (Meredith, New York, 1966).

7 M. B. Sachs and N. Y. S. Kiang, J. Acoust. Soc. Amer. 43, 1120 (1968); R. Klinke, G. Boerger, S. Gruber, Pfluegers Arch. Ges. Physiol. 306, 165 (1969)

8 Supported in part by PHS grant MH 15828. I thank Drs. A. D. Baddeley, R. Erickson, and D. M. Green for their interesting discussions.

27 September 1971; revised 19 November 1971 